Pacific Journal of Mathematics

THE COHOMOLOGICAL DESCRIPTION OF A TORUS ACTION 


\section{THE COHOMOLOGICAL DESCRIPTION OF A TORUS ACTION}

\section{DAVID GoLBer}

The theorem proved in this paper is an example of a "regularity" theorem in the study of topological group actions-that is, it shows that a general topological action of a group continues to have certain properties of "linear" actions. Consider an action of a torus $T$ on a cohomology $n$-sphere $X$, with fixed point set the cohomology $r$-sphere $F$. Consider the map $H^{n}\left(X_{T} ; Z\right) \rightarrow H^{n}\left(F_{T} ; Z\right)$, and let $c \eta$ be the image of the generator of $H^{n}(X ; Z)$, considered as lying in $H^{n-r}(B T ; Z)$, where $c$ is an integer and $\eta$ has no nontrivial integer divisors. The polynomial part $\eta$ is well understood. The theorem will evaluate the integer part $c$ in the following sense: in the linear case, $c$ can be easily expressed in terms of the dimensions of the fixed point sets of various nonconnected subgroups of $T$. It is shown that this formula continues to hold in the general topological case, given some weak assumptions. There is also a corresponding result for the case $F=\varnothing$.

The main tool will be the fibration $\pi: X_{T} \rightarrow B_{T} \equiv B T$, where $X_{T}$ is as usual $E_{T} \times{ }_{T} X$. We will use the usual limit arguments to allow ourselves to pretend that $E_{T}$ is compact. Cohomology will be sheaf cohomology with compact supports (which will not usually be indicated). The spectral sequence of $X_{T} \rightarrow B T$ with coefficients in $A$ will be denoted $E_{r}\left(X_{T} ; A\right)$. The fixed point set of $T$ acting on $X$ will be denoted $F(T, X) \equiv F(T) . \quad X \sim_{z} Y\left(X \sim_{p} Y\right)$ will mean that $X$ is a compact $Z$-cohomology $\left(Z_{p}\right.$-cohomology) manifold with $Z\left(Z_{p}\right)$ cohomology ring the same as that of $Y . \operatorname{dim}_{p}(X)$ or $\operatorname{dim}_{Z}(X)$ will be the usual cohomological dimension of $X$ over $Z_{p}$ or $Z$. See [1] or [2] for details. For an abelian group $A$, let $\mathscr{F} A$ be $A / \operatorname{Torsion}(A)$.

If a torus $T$ acts on a space $X$, a subtorus $H$ of $T$ is said to be distinguished if $F(H) \supseteqq F(K)$ for any subtorus $K$ which has $K \supseteqq H$. In particular, the distinguished corank one subtori of $T$ are those subtori $H$ of corank one in $T$ that have $F(H) \supsetneq F(T)$. Recall that given a corank one subtorus of $T$, there is a corresponding integer-valued linear functional on the Lie algebra of $T$, a corresponding element of $H^{1}(T ; Z)$ and a corresponding element (not divisible by any integer) in $H^{2}(B T ; Z)$.

Now consider a torus $T$ acting on $X \sim{ }_{2} S^{n}$. Let $F(T) \sim{ }_{2} S^{r}$, and look at $F_{T} \subseteq X_{T}$. Consider the cases $r>0, r=0$, and $r=-1(F(T)=\varnothing)$ separately. 
In case $r>0$, the map

$$
H^{n}(X ; Z) \cong E_{\infty}^{0, n}\left(X_{T} ; Z\right) \longrightarrow E_{\infty}^{n-r, r}\left(F_{T} ; Z\right) \cong H^{n-r}(B T ; Z)
$$

takes the generator of $H^{n}(X ; Z)$ to $c \eta$ where $c$ is an integer, and $\eta$ is $\Pi g_{i}^{\left(n_{i}-r\right) / 2}$. (Here the $g_{i}$ 's correspond to the distinguished corank one subtori $U_{i}$ of $T$, and $n_{i}=\operatorname{dim}_{z} F\left(U_{i}\right)$.)

In case $r=0, F(T) \sim{ }_{Z} S^{0}$, we have $\pi: F_{T} \cong F \times B_{T} \rightarrow B T$, and the inclusion $F_{T} \rightarrow X_{T}$ induces

$$
H^{n}(X ; Z) \longrightarrow \widetilde{H}^{0}(F ; Z) \otimes H^{n-r}(B T ; Z)
$$

which takes $g$ to (generator) $\otimes c \eta$ as before.

In case $r=-1, F(T)=\varnothing$, the transgression

$$
H^{n}(X ; Z) \longrightarrow H^{n+1}(B T ; Z)
$$

takes the generator to $\mathrm{c} \eta$.

The theorem below will identify the integer $c$.

Let $p$ be any prime. (Several of the objects below will depend on $p$, although this dependence will not be explicitly indicated.) (The letter $p$ will also be used as one of indices of a spectral sequence, but hopefully no confusion will result.) For $i=1,2, \cdots$ let $S(i)$ be the subgroup of elements $t$ of $T$ such that $p^{i} t=1$, the identity element of $T$. Let $S(0)$ be the subgroup of $T$ consisting of 1 only. Clearly $S(i) \cong\left(Z_{p^{i}}\right)^{k}$, where $k$ is the rank of $T$. Each $F(S(i))$ is a $Z_{p}$-cohomology $n_{i}$-sphere for some $n_{i}$.

THEOREM. Suppose that for any prime $p$ and $i=1,2, \cdots$ that $F(S(i))$ has finitely generated Z-cohomology. Let $p^{a}$ be the largest power of $p$ that divides $c$. Then

$$
\sum_{i=1}^{\infty}\left[\operatorname{dim}_{p} F(S(i))-\operatorname{dim}_{z} F\right]=2 a .
$$

Further, $F(S(i))=F$ for $i>a$.

Proof. The second claim follows from the first and the fact that $\operatorname{dim}_{p} F(S(i))-\operatorname{dim}_{z} F$ is always even. (See [1], Chapter IV.)

We will first do the case $r>0$ and then reduce the other two cases to this case.

Consider the spectral sequence of $F(S(i))_{T} \rightarrow B T$. Because $F(S(i)) \sim{ }_{p} S^{n_{i}}$ and has finitely generated integral cohomology, it is easy to see from the universal coefficient theorem that $H^{*}(F(S(i)) ; Z)$ has no $p$-torsion, that $H^{\circ}(F(S(i)) ; Z)=Z$, and that $\mathscr{F} H^{*}(F(S(i)) ; Z)=$ $H^{*}\left(S^{n} ; Z\right)$. Because $r>0$, the $Z_{p}$ spectral sequence of $F(S(i))_{T} \rightarrow B_{T}$ 
collapses. It is then easy to verify the following facts about $E_{\infty}\left(F(S(i))_{T} ; Z\right)$ :

(i) $E_{\infty}\left(F(S(i))_{T} ; Z\right)$ has no $p$-torsion.

(ii) $\mathscr{F} E_{\infty}^{p, q}\left(F(S(i))_{T} ; Z\right) \cong H^{p}(B T ; Z)$ if $q=0$ or $n_{i}$, and $=0$ otherwise. (As abelian groups with no reference to the multiplicative structure).

(iii) The bottom row $E_{\infty}^{*, 0}\left(F(S(i))_{T} ; Z\right)$ is isomorphic to $H^{*}(B T ; Z)$, as a ring.

(iv) Let $h$ be a generator of $\mathscr{F} E_{\infty}^{0, n_{i}}\left(F(S(i))_{T} ; Z\right)$. Multiplication by $h$ defines a map

$$
H^{p}(B T ; Z) \cong E_{\infty}^{p, 0}\left(F(S(i))_{T} ; Z\right) \longrightarrow \mathscr{F} E_{\infty}^{p, n_{i}}\left(F(S(i))_{T} ; Z\right) \cong H^{p}(B T ; Z)
$$

This map is monomorphism, and its cokernel is finite with non- $p$ order.

We know $F(S(i)) \sim{ }_{p} S^{n_{i}}$ for $i=0,1,2, \cdots$ where $n_{0}=n$, and $F(S(\ell+1))=F$ for $\ell$ large enough, so $n_{\ell+1}=r$. We have

$$
X=F(S(0)) \supseteqq F(S(1)) \supseteqq \cdots \supseteqq F(S(\ell)) \supseteqq F(S(\ell+1))=F .
$$

We can consider the inclusion map $F_{T} \rightarrow X_{T}$ to be the composition

$$
F(S(\ell+1))_{T} \longrightarrow F(S(\ell))_{T} \longrightarrow \cdots \longrightarrow F(S(1))_{T} \longrightarrow F(S(0))_{T} .
$$

Let $h_{i}$ be a generator of $\mathscr{F} E_{\infty}^{0, n_{i}}\left(F(S(i))_{T} ; Z\right)$. We will show below that the induced map $\varphi: \mathscr{F} E_{\infty}\left(F(S(i))_{T} ; Z\right) \rightarrow \mathscr{F} E_{\infty}\left(F(S(i+1))_{T} ; Z\right)$ has $\varphi\left(h_{i}\right)$ divisible by $p^{i\left(n_{i}-n_{i+1}\right) / 2}$ and by no higher power of $p$. Using this and the facts (i)-(iv), we can show that $2 a=\sum_{i=0}^{\ell} i\left(n_{i}-n_{i+1}\right)$, which equals $\sum_{i=1}^{\ell}\left(n_{i}-n_{\ell+1}\right)$, which is our conclusion. Thus we only have to prove our claim about the number of factors of $p$ dividing $\varphi\left(h_{i}\right)$.

Consider the following diagram:

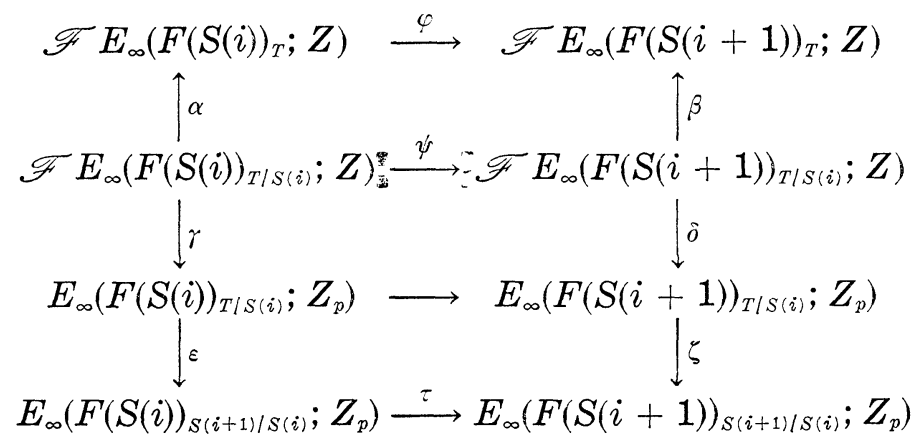

Let $k_{i}$ be the generator of $\mathscr{F} E_{\infty}^{0, n_{i}}\left(F(S(i))_{T / S(i)} ; Z\right)$. It is easy to see that $\alpha\left(k_{i}\right)$ is a non-p multiple of $h_{i}$. Now the map $\beta$ on the 
$E^{2,0}$ terms is $Z^{k} \cong H^{2}(B T / S(i) ; Z) \rightarrow H^{2}(B T ; Z) \cong Z^{k}$, which is multiplication by $p^{i}$. Since $\psi\left(k_{i}\right)$ lies in filtration degree $n_{i}-n_{i+1}$, we can see using (iv) above that $\beta\left(\psi\left(k_{i}\right)\right)$ contains precisely $i\left(n_{i}-n_{i+1}\right) / 2$ more factors of $p$ than $\psi\left(k_{i}\right)$ does. Thus it is sufficient to show that $\psi\left(k_{i}\right)$ is not divisible by $p$.

The map $\delta$ is reduction $\bmod p$, so it suffices to show that $\delta \psi\left(k_{i}\right) \neq 0$. Therefore it suffices to show that $\tau \varepsilon \gamma\left(k_{i}\right) \neq 0$. But $S(i+1) / S(i) \cong$ $\left(Z_{p}\right)^{k}$ acts on $F(S(i)) \sim{ }_{p} S^{n_{i}}$, with fixed point set $F(S(i+1)) \sim{ }_{p} S^{n_{i+1}}$, and $\varepsilon \gamma\left(k_{i}\right)$ is the generator of $E_{\infty}^{0, n_{i}}\left(F(S(i))_{S(i+1) / S(i)} ; Z_{p}\right)$. In these circumstances, we must have $\tau \varepsilon \gamma\left(k_{i}\right)$ nonzero (see [1], Chapter XIII, and [3]) which finishes the proof in the case $r>0$.

The cases $r=0$ and $r=-1$ are handled by replacing $X$ by $S X$ and $S^{2} X$ respectively, where $S X$ denotes the nonreduced suspension. The action of $T$ on $S X$ (or $S^{2} X$ ) then has a nonempty connected fixed point set, so the problem is reduced to the previous case. It is not hard to see that if $X \sim{ }_{z} S^{n}$, then $S X \sim{ }_{Z} S^{n+1}$. Thus we only need to show: (1) Suppose $T$ acts on $X \sim{ }_{2} S^{n}$ with $F(T)=\varnothing$. Consider the actions of $T$ on $X(F(T, X)=\varnothing, r=-1)$ and on $S X\left(F(T, S X) \sim{ }_{z} S^{0}, r=0\right)$. One gets an integer $c$ from each action. We need to show that the two $c$ 's are the same, at least up to sign. And (2) Suppose $T$ acts on $X \sim{ }_{z} S^{n}$ with $F(T) \sim{ }_{z} S^{0}$. Consider the actions of $T$ on $X\left(F(T, X) \sim{ }_{z} S^{0}, r=0\right)$ and on $S X\left(F(T ; S X) \sim{ }_{z} S^{1}\right.$, $r=1$ ). Again, one gets two $c$ 's which we need to prove are the same up to sign.

The second case, going from $r=0$ to $r=1$, is easy; one merely uses the naturality of the suspension map.

In the first case, going from $r=-1$ to $r=0$, we have an action of $T$ on $X \sim{ }_{z} S^{n}$ with $F=\varnothing$, so $n$ is odd. In the spectral sequence of $p: X_{T} \rightarrow B_{T}$, the generator of $H^{n}(X)$ transgresses to $c \eta \in H^{n+1}(B T), c \eta \neq 0$. On the other hand, the spectral sequence of $q:(S X)_{T} \rightarrow B T$ collapses. Then it is easy to check that $H^{n}\left(X_{T}\right)=0$, $H^{n+1}\left(X_{T}\right)=H^{n+1}(B T) /\langle c \eta\rangle, \quad H^{n+2}\left((S X)_{T}\right)=0$, and there is a split short exact sequence

$$
0 \longrightarrow H^{n+1}(B T) \stackrel{q^{*}}{\longrightarrow} H^{n+1}\left((S X)_{T}\right) \stackrel{j^{*}}{\longrightarrow} H^{n+1}(S X) \longrightarrow 0,
$$

where $j: S X \rightarrow(S X)_{T}$ is the inclusion of the fiber. One can consider $(S X)_{T}$ to be the space $\left(X_{T} \times I\right) / \sim$, where $(a, 1) \sim(b, 1)$ and $(a, 0) \sim$ $(b, 0)$ iff $p(a)=p(b)$. The map $q:(S X)_{T} \rightarrow B T$ is given by $[a, t] \rightarrow p(a)$. The sets $E_{0}=\left\{[a, 0] \in(S X)_{T}\right\}$ and $E_{1}=\left\{[a, 1] \in(S X)_{T}\right\}$ are each mapped homeomorphically onto $B T$ by $q$. Let $i_{0}$ and $i_{1}: B T \rightarrow(S X)_{T}$ be the corresponding two sections of $q$. Showing that the action on $S X$ gives rise to the same $c$ as that on $X$ reduces to showing that in 


$$
H^{n+1}(S X) \stackrel{j^{*}}{\longleftarrow} H^{n+1}\left((S X)_{T}\right) \stackrel{i_{0}^{*}-i_{1}^{*}}{\longrightarrow} H^{n+1}(B T),
$$

$\left(i_{0}^{*}-i_{1}^{*}\right) \circ\left(j^{*}\right)^{-1}$ takes the generator of $H^{n+1}(S X)$ to $\pm c \eta$.

Let $U=\left\{[a, t] \in(S X)_{T} \mid t>1 / 4\right\}$; let $V=\left\{[a, t] \in(S X)_{T} \mid t<3 / 4\right\}$. $U$ and $V$ have $E_{0}$ and $E_{1}$ as strong deformation retracts, $U \cup V=$ $(S X)_{T}$, and $U \cap V$ has $\left\{[a, 1 / 2] \in(S X)_{T}\right\} \cong X_{T}$ as a strong deformation retract. Consider the Meyer-Vietoris sequence of pair $(U, V)$ :

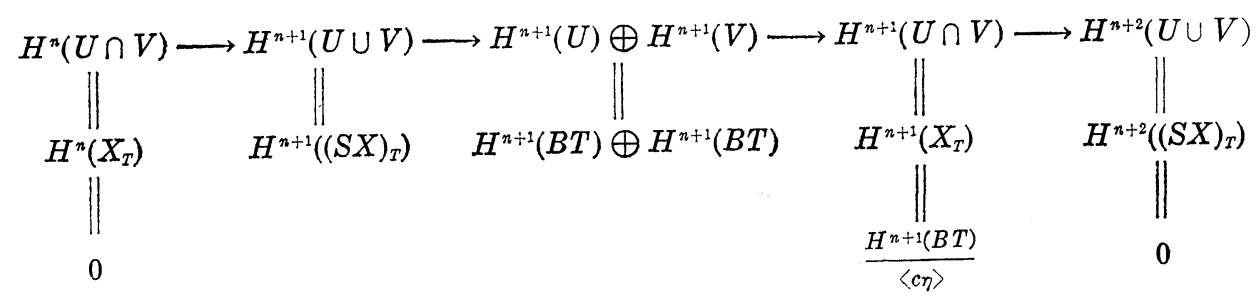

so we have the commutative diagram

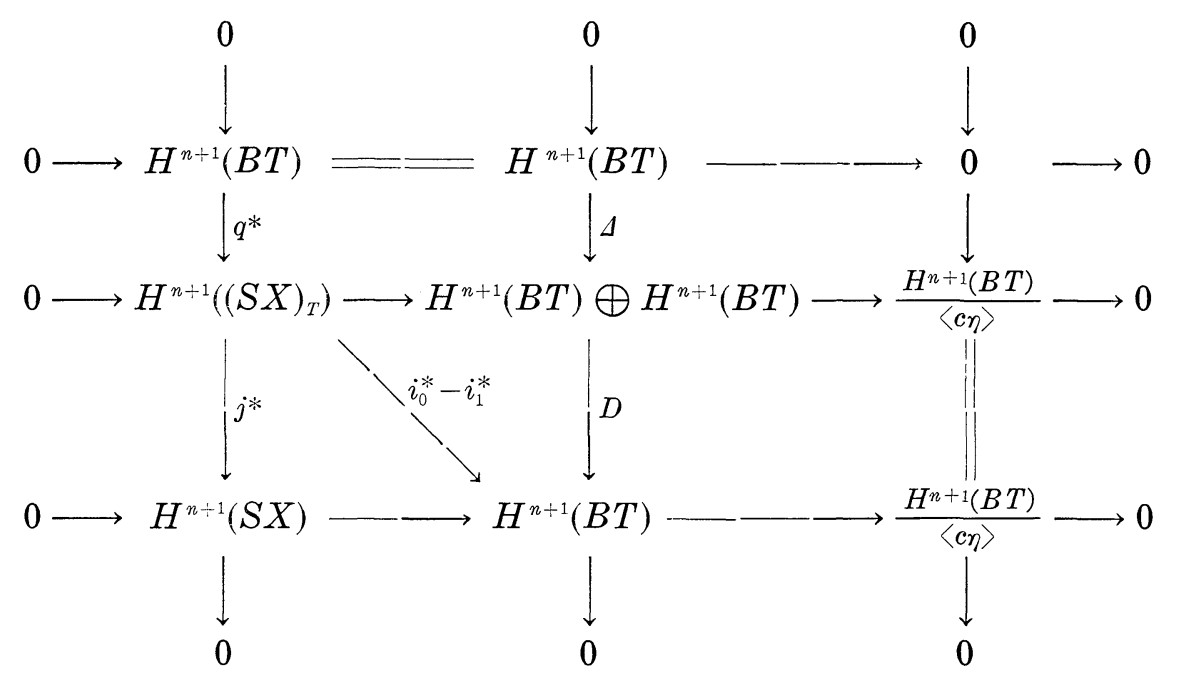

where $\Delta$ is the diagonal map and $D$ is given by $D(a, b)=a-b$. The top two rows and all three columns are exact, so that bottom row is also exact. The horizontal map on the bottom left is $\left(i_{0}^{*}-i_{1}^{*}\right) \circ\left(j^{*}\right)^{-1}$, so we can see that this map takes the generator of $H^{n+1}(S X)$ to $\pm c \eta \in H^{n+1}(B T)$, as was to be shown.

This finishes the proof of the theorem.

\section{REFERENCES}

1. A. Borel, et al., Seminar on Transformation Groups, Ann. of Math. Studies No. 46, Princeton University Press, 1960.

2. G. Bredon, Sheaf Theory, McGraw-Hill, New York, 1967. 
3. D. Golber, Torus actions on a product of two odd spheres, Topology, 10 (1971), 313-316.

4. Wu-Yi Hsiang, On Generalizations of a Theorem of A. Borel and Their Applications in the Study of Topological Actions, Topology of Manifolds, Cantrell and Edwards, editors, Markham Publishing Co., Chicago, 1970, pp. 276-290.

Received December 7, 1971.

The University of California, San Diego 


\section{PACIFIC JOURNAL OF MATHEMATICS}

\section{EDITORS}

D. Gilbarg and J. Milgram Stanford University

Stanford, California 94305

R. A. Beaumont

University of Washington

Seattle, Washington 98105
J. DugundJI

Department of Mathematics

University of Southern California

Los Angeles, California 90007

RICHARD ARENS

University of California

Los Angeles, California 90024

ASSOCIATE EDITORS
E. F. BECKENBACH
B. H. NeumanN
F. WOLF
K. YoSHIDA

\section{SUPPORTING INSTITUTIONS}

UNIVERSITY OF BRITISH COLUMBIA

CALIFORNIA INSTITUTE OF TECHNOLOGY

UNIVERSITY OF CALIFORNIA

MONTANA STATE UNIVERSITY

UNIVERSITY OF NEVADA

NEW MEXICO STATE UNIVERSITY

OREGON STATE UNIVERSITY

UNIVERSITY OF OREGON

OSAKA UNIVERSITY
UNIVERSITY OF SOUTHERN CALIFORNIA

STANFORD UNIVERSITY

UNIVERSITY OF TOKYO

UNIVERSITY OF UTAH

WASHINGTON STATE UNIVERSITY

UNIVERSITY OF WASHINGTON

$\stackrel{*}{*} \stackrel{*}{*} \stackrel{*}{*}$ AMERICAN MATHEMATICAL SOCIETY

NAVAL WEAPONS CENTER 


\section{Pacific Journal of Mathematics}

\section{Vol. 46, No. $1 \quad$ November, 1973}

Allan Francis Abrahamse, Uniform integrability of derivatives on $\sigma$-lattices .......................................... 1

Ronald Alter and K. K. Kubota, The diophantine equation $x^{2}+D=p^{n} \ldots \ldots \quad 11$

Grahame Bennett, Some inclusion theorems for sequence spaces .......... 17

William Cutler, On extending isotopies ........................ 31

Robert Jay Daverman, Factored codimension one cells in Euclidean

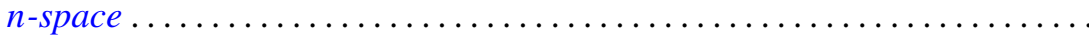

Patrick Barry Eberlein and Barrett O’Neill, Visibility manifolds ............ 45

M. Edelstein, Concerning dentability .......................... 111

Edward Graham Evans, Jr., Krull-Schmidt and cancellation over local

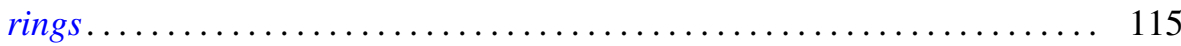

C. D. Feustel, A generalization of Kneser's conjecture ................ 123

Avner Friedman, Uniqueness for the Cauchy problem for degenerate parabolic equations .......................................... 131

David Golber, The cohomological description of a torus action ............ 149

Alain Goullet de Rugy, Un théorème du genre "Andô-Edwards" pour les

Fréchet ordonnés normaux..............................

Louise Hay, The class of recursively enumerable subsets of a recursively enumerable set ........................................

John Paul Helm, Albert Ronald da Silva Meyer and Paul Ruel Young, On orders of translations and enumerations...

Julien O. Hennefeld, A decomposition for $B(X)^{*}$ and unique Hahn-Banach

extensions

Gordon G. Johnson, Moment sequences in Hilbert space .

Thomas Rollin Kramer, A note on countably subparacompact spaces ...

Yves A. Lequain, Differential simplicity and extensions of a derivation ....

Peter Lorimer, A property of the groups Aut $\mathrm{PU}\left(3, q^{2}\right) \ldots$

225

Yasou Matsugu, The Levi problem for a product manifold.

231

John M.F. O'Connell, Real parts of uniform algebras ......

William Lindall Paschke, A factorable Banach algebra without bounded

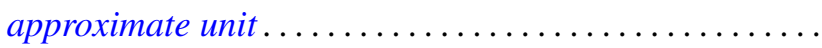

Ronald Joel Rudman, On the fundamental unit of a purely cubic field ....

Tsuan Wu Ting, Torsional rigidities in the elastic-plastic torsion of simply connected cylindrical bars .........................

Philip C. Tonne, Matrix representations for linear transformations on analytic sequences...................................

Jung-Hsien Tsai, On E-compact spaces and generalizations of perfect

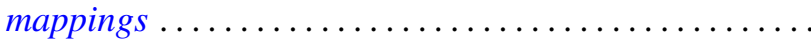

Alfons Van Daele, The upper envelope of invariant functionals majorized by an invariant weight. .. 